Sonderdruck aus

Hoppe-Seyler's Zeitschrift für Physiologische Chemie

Walter de Gruyter \& Co., Berlin 30

Hoppe-Seyler's Z. Physiol. Chem.

Bd. 353, S. 1638 - 1645, Oktober 1972

\title{
Eigenschaften des Trypsin-ähnlichen Enzyms (Akrosin) aus Eberspermien
}

Hans Schießler*, Hans Fritz*, Marianne Arnhold*, Edwin Fink ${ }^{* *}$ und Harald Tschesche***

(Der Schriftleitung zugegangen am 17. August 1972)

Herrn Professor Dr. Dr. Eugen Werle zum 70. Geburtstag gewidmet 
Zusammenfassung: 1. Für die enzymatische Bestimmung von Eberakrosin eignen sich die synthetischen Trypsinsubstrate BLNA (pH-Optimum 8,0, Michaeliskonstante $\left.K_{m}=0,65 \mathrm{mM}\right)$, BAPA $(\mathrm{pH}-$ Optimum 7,8, Michaeliskonstante $K_{m}=0,39 \mathrm{mM}$ ) und BAEE (bestimmt im kombinierten Testsystem mit Hilfe von $\mathrm{ADH} / \mathrm{NAD}^{\oplus}$ ). Die zum Nachweis benötigten molaren Akrosinkonzentrationen liegen beim BAEE/ADH-System bei $10^{-10} \mathrm{M}$, für BAPA bei $10^{-9} \mathrm{M}$ und für BLNA bei $10^{-8} \mathrm{M}$. Die Endoproteinaseaktivität des Akrosins ist durch die nachgewiesene Spaltung des Proteinsubstrates Azocasein belegt. BTEE wird durch Eberakrosin nicht hydrolysiert.

2. Eberakrosin von höherem Reinheitsgrad wird besonders aus neutralen bis leicht alkalischen Lö- sungen effektiv an Glasoberflächen adsorbiert und inaktiviert, so daß bei allen Manipulationen ausschließlich Plastikgeräte bzw. silikonisierte Glasgeräte zu verwenden sind.

3. Die bimolekulare Geschwindigkeitskonstante für die Hemmung des Akrosins durch DFP wurde zu $630 \mathrm{l} \times \mathrm{Mol}^{-1} \times \mathrm{min}^{-1}$ bestimmt, für die Hemmung durch TLCK zu $23 l \times \mathrm{Mol}^{-1} \times \mathrm{min}^{-1}$.

4. Die bei Titration von Eberakrosin mit NPGB und Bdellin B-3 gefundenen molaren Akrosinkonzentrationen zeigen eine gute Übereinstimmung.

5. Um die Hemmwirkung von Inhibitoren mit geringerer Affinität zum Akrosin (z. B. Trypsininhibitor II aus Humansperma, Inhibitoren aus Seeanemonen, Benzamidin) demonstrieren zu können, eignet sich besonders BLNA als Substrat.

\section{Properties of the trypsin-like enzyme (acrosin) from boar spermatozoa}

Summary: 1) The synthetic trypsin substrates BLNA (pH optimum 8.0, $K_{m}=0.65 \mathrm{~mm}$ ), BAPA (pH optimum 7.8, $K_{m}=0.39 \mathrm{mM}$ ) and BAEE (estimated in the combined test system with $\mathrm{ADH} / \mathrm{NAD}^{\oplus}$ ) are suitable substrates for the estimation of boar acrosin as well. The following molar acrosin concentrations are necessary for the different estimation systems: BAEE/ADH $10^{-10} \mathrm{M}$, BAPA $10^{-9} \mathrm{M}$, BLNA $10^{-8} \mathrm{M}$. The endoproteinase activity of boar acrosin is demonstrated by its action on azocasein. BTEE is not hydrolyzed by boar acrosin.

2) Purified boar acrosin is effectively adsorbed and
* Priv.-Doz. Dr. H. Fritz und Dipl.-Chem. H. Schießler, Institut für Klinische Chemie und Klinische Biochemie der Universität München, D-8 München 2, Nußbaumstraße 20.

** Dr. E. Fink, z. Zt. Brookhaven National Laboratory, Upton, L. I., N. Y. 11973 , USA.

*** Doz. Dr. H. Tschesche, Technische Universität München, Lehrstuhl für Organische Chemie und Biochemie, D-8 München 2, Arcisstraße 21.

\section{Abkürzungen:}

$\mathrm{ADH}=$ Alkohol-Dehydrogenase (EC 1.1.1.1)

BAEE $=N^{\alpha}$-Benzoyl-L-arginin-äthylester

BAPA $=N^{\alpha}$-Benzoyl-DL-arginin-p-nitroanilid

BLNA $=N^{\alpha}$-Benzoyl-L-lysin-p-nitroanilid

BTEE $=N^{\alpha}$-Benzoyl-L-tyrosin-äthylester

DFP $=$ Diisopropyl-fluorophosphat

NPGB = 4-Guanidinobenzoesäure-4-nitrophenylester

TLCK $=N^{\alpha}$-Tosyl-L-lysyl-chlormethan

TPCK $=N^{\alpha}$-Tosyl-L-phenylalanyl-chlormethan 
inactivated on glass surfaces. Therefore, all manipulations were done in plastic apparatures or siliconized glass ware.

3) The bimolecular velocity constant for the inhibition of acrosin by DFP was found to be $630 l \times$ $\mathrm{mol}^{-1} \times \mathrm{min}^{-1}$ and for the inhibition by TLCK $23 l \times \mathrm{mol}^{-1} \times \mathrm{min}^{-1}$.

4) Molar acrosin concentrations estimated by titration with NPGB and the trypsin-plasmin inhibitor Bdellin B-3 from leeches are in good agreement.

5) BLNA is a suitable substrate for titration experiments in which inhibitors with lower affinities to boar acrosin (trypsin inhibitor II from human sperm, inhibitor from sea anemones, benzamidine) are used.
Der Nachweis einer engeren Verwandtschaft zwischen einer akrosomalen Spermien-Proteinase und dem Pankreasenzym Trypsin wurde erstmals auf einer breiteren Basis von Zaneveld und Mitarb. $[1,2]$ erbracht. Sie verwendeten für ihre mehr qualitativen Vergleichsuntersuchungen synthetische Estersubstrate und irreversible Inhibitoren für Trypsin, ein Proteinsubstrat sowie eine Reihe von Trypsininhibitoren natürlichen Ursprungs. Vergleichende Untersuchungen mit ähnlichen Ergebnissen liegen inzwischen auch für die akrosomale Proteinase aus menschlichen Spermien ${ }^{[3]}$ vor. In dieser Arbeit werden einige enzymatische Eigenschaften des durch Affinitätschromatographie isolierten Eberakrosins $^{[4]}$ quantitativ erfaßt.

\section{Material und Methodik}

\section{Verwendete Substanzen}

Alkohol-Dehydrogenase aus Hefe (Art. 15418) von Boehringer Mannheim GmbH; Azocasein (Art. 11610) von Fluka AG Buchs; Benzamidin-HCl (Art. B-200-4) von Aldriche-Europe; $N^{\alpha}$-Benzoyl-L-arginin-äthylester$\mathrm{HCl}$ (Art. 1672), $N^{\alpha}$-Benzoyl-DL-arginin-4-nitroanilid$\mathrm{HCl}$ (Art. 1670), $N^{\alpha}$-Benzoyl-L-lysin-4-nitroanilid-HCl (Art. 10269) von E. Merck Darmstadt; $N^{\alpha}$-Benzoyl-Ltyrosin-äthylester (Art. 13110) von Fluka AG Buchs; Diisopropyl-fluorophosphat von Bayer AG, Werk Elberfeld; 4-Guanidinobenzoesäure-4-nitrophenylester$\mathrm{HCl}$ (Art. 10562) von E. Merck Darmstadt; $\beta$-Nicotinamid-adenin-dinucleotid (Art. 15300) von Boehringer Mannheim GmbH; $N^{\alpha}$-Tosyl-L-lysyl-chlormethan- $\mathrm{HCl}$ (Art. 8163) von E. Merck Darmstadt; Triäthanolamin-

1 Zaneveld, L. J. D., Polakoski, K. L., Robertson, R. T. \& Williams, W. L. (1971) in Proc. Int. Res. Conf. on Proteinase Inhibitors (Fritz, H. \& Tschesche, H., Hrsg.) S. 236-244, Walter de Gruyter, Berlin.

2 Zaneveld, L. J. D., Polakoski, K. L. \& Williams, W. L. (1972) Biol. Reprod. 6, 30-39.

3 Zaneveld, L. J. D., Dragoje, B. M. \& Schumacher, G. F. B. (1972) Science, 177, 702-703.

${ }^{4}$ Fink, E., Schießler, H., Arnhold, M. \& Fritz, H. (1972) diese Z. 353, 1633-1637, vorstehend.
HCl p. A. (Art. 8379) von E. Merck Darmstadt. Alle weiteren Reagentien waren vom höchsten im Handel erhältlichen Reinheitsgrad.

Proteaseinhibitoren: Trypsin-Plasmin-Kallikrein-Chymotrypsin-Inhibitor aus Seeanemonen ${ }^{[5]}, 3,3$ IU (für Trypsin)/mg (Gemisch der Isoinhibitoren); Trypsininhibitor II aus Humansperma ${ }^{[6]}, 2,17 \mathrm{IU} / \mathrm{mg}$.

\section{Aktivitätsbestimmungen mit verschiedenen Substraten}

Die Herstellung von Verdünnungen erfolgte nur in Plastikgefäßen bzw. mit Plastikpipetten (Mikrolitersystem Eppendorf). Die zu den Messungen verwendeten Glasküvetten wurden jeweils nach eintägigem Gebrauch neu silikonisiert. Die Mischung der Reagentien in den Küvetten erfolgte nach Verschluß mit einem Teflondeckplättchen durch mehrmaliges Umwenden.

1. BAPA: Die Messung der Akrosinaktivität mit Hilfe von BAPA erfolgte nach der für die Trypsinaktivitätsbestimmung angegebenen Vorschrift ${ }^{[7]}$. Reihenfolge der Reagentienzugabe in die Meßküvette: $1,0 \mathrm{ml}(1 \mathrm{mg})$ BAPA-Lösung plus (2-x) $\mathrm{m} l 0,2 \mathrm{M}$ Triäthanolamin- $\mathrm{HCl}$, pH 7,8 (Testpuffer) plus $\mathrm{x} \mathrm{ml}$ Akrosinlösung. Die Extinktionsänderung bei $405 \mathrm{~nm}$ wurde $10 \mathrm{~min}$ lang in min-Intervallen registriert. Meßtemperatur: $25^{\circ} \mathrm{C}$.

2. BLNA: Die Substratlösung enthielt $2,4 \mathrm{mg} B L N A / \mathrm{m} l$ dest. Wasser. Methodik analog dem BAPA-Test.

3. BAEE: Das bei der BAEE-Spaltung freigesetzte Äthanol wurde mit Hilfe von $\mathrm{ADH}$ und $\mathrm{NAD}^{\oplus}$ im kombinierten Test bestimmt ${ }^{[8]}$. Bedingungen und Reagentien s. 1.c. ${ }^{[9]}$. Die Extinktionsänderung bei $366 \mathrm{~nm}$ wurde $10 \mathrm{~min}$ lang in min-Intervallen registriert. Meßtemperatur: $25^{\circ} \mathrm{C}, \mathrm{pH}$ in der Küvette: 8,7 .

${ }^{5}$ Fritz, H., Brey, B. \& Béress, L. (1972) diese Z. 353. 19-30.

${ }^{6}$ Fink, E., Jaumann, E., Fritz, H., Ingrisch, H. \& Werle, E. (1971) diese Z. 352, 1591 - 1594.

${ }^{7}$ Fritz, H., Trautschold, I. \& Werle, E. (1970) in Methoden d. Enzymat. Analyse (Bergmeyer, H. U., Hrsg.) S. 1021-1038, Verlag Chemie, Weinheim/ Bergstr.

8 Trautschold, I., Werle, E. \& Schweitzer, G. (1970) in 1. c. [7], S. $1011-1020$ (,,Manuelles Verfahren“, S. 1012f.).

9 Fritz, H., Eckert, I. \& Werle, E. (1967) diese Z. 348. 1120-1132. 
4. Azocasein: Die für die Trypsinbestimmung mit Azocasein als Substrat angegebene Vorschrift in $1 . c^{[7]}$ wurde angewandt. Inkubationstemperatur: $30^{\circ} \mathrm{C}$. Aktueller pH-Wert : 7,6.

\section{Titration von Akrosin mit Inhibitoren}

1. Proteaseinhibitoren: Zur Methodik s. Vorschrift in 1. c. ${ }^{[10]}$.

2. DFP: $0,5 \mu \mathrm{Mol}$ DFP wurden mit ca. $2 \mathrm{nMol}$ (berechnet aus der Titration mit Bdellin B-3[11]) Akrosin in $10 \mathrm{ml} 0,1 \mathrm{M}$ Triäthanolamin- $\mathrm{HCl}, \mathrm{pH} 7,8$, bei $25^{\circ} \mathrm{C}$ in einem Plastikgefäß inkubiert. Nach den in Abb. 7 angegebenen Inkubationszeiten wurde jeweils $1 \mathrm{~m} l$ der Reaktionslösung entnommen und die darin vorhandene Akrosinaktivität nach Zugabe von $1,0 \mathrm{~m} l$ des Testpuffers mit Hilfe des Substrates BLNA bestimmt.

3. TLCK: $2,0 \mu \mathrm{Mol}$ TLCK wurden mit ca. $2 \mathrm{nMol}$ Akrosin in $5 \mathrm{ml} 0,1 \mathrm{M}$ Triäthanolamin- $\mathrm{HCl}, \mathrm{pH} 6,9$, bei $25^{\circ} \mathrm{C}$ in einem Plastikgefäß inkubiert. Nach den in Abb. 7 angegebenen Inkubationszeiten wurden jeweils $0,5 \mathrm{ml}$ der Reaktionslösung entnommen und die darin vorhandene Akrosinaktivität nach Zugabe von $1,5 \mathrm{ml}$ des Testpuffers mit Hilfe des BLNA-Substrates bestimmt.

4. PNGB: Die Titration von Akrosin mit PNGB erfolgte nach der Vorschrift von Chase und Shaw ${ }^{[12]}$. Die eingesetzten $0,5 \mathrm{~m} /$ Enzymlösung enthielten 1,07 U (BAPA) Akrosin.

\section{Ergebnisse}

Für die folgenden Untersuchungen wurde das mit Hilfe der Affinitätschromatographie isolierte Akro$\sin ^{[4]}$ mit einer spezifischen Aktivität von $5 \mathrm{U}$ (BAPA)/mg Protein verwendet.

\section{Synthetische Substrate}

Aus den Abb. 1, 2 und 3 sind die Meßbereiche ersichtlich, innerhalb derer die Spaltungsgeschwindigkeit der Substrate BAPA, BLNA und BAEE gemessen anhand der Extinktionsänderung pro

Abb. 2. Spaltung von BLNA durch Eberakrosin.

Ordinate, Abszisse, Bedingungen: s. Legende von Abb.1.

10 Fritz, H., Förg-Brey, B., Fink, E,. Schießler, H., Jaumann, E. \& Arnhold, M. (1972) diese Z. 353, 1007 $-1009$.

11 Fritz, H., Förg-Brey, B., Schießler, H., Arnhold, M. \& Fink, E. (1972) diese Z. 353, 1010-1012.

12 Chase, T., Jr. \& Shaw, E. (1970) Methods Enzymol. 19, 20-27.

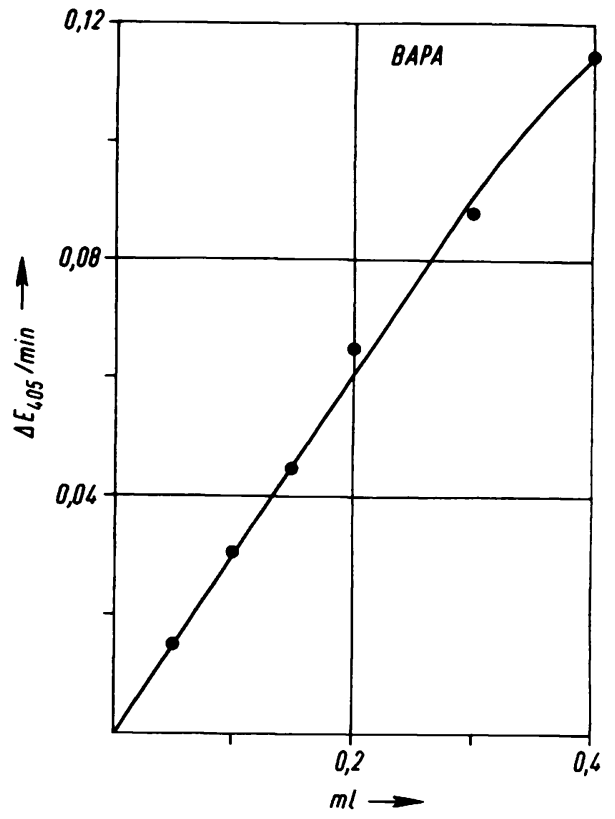

Abb. 1. Spaltung von BAPA durch Eberakrosin.

Ordinate: Extinktionsänderung bei $405 \mathrm{~nm}$ pro min. Abszisse: Eingesetzte Akrosinmenge in $\mathrm{ml}$. Die experimentellen Bedingungen sind in ,,Material und Methodik" beschrieben. Die auf den Abszissen angegebenen $\mathrm{ml-Mengen} \mathrm{in} \mathrm{den} \mathrm{Abb.} \mathrm{1-3} \mathrm{stehen} \mathrm{in} \mathrm{keiner} \mathrm{Be}$ ziehung zueinander.

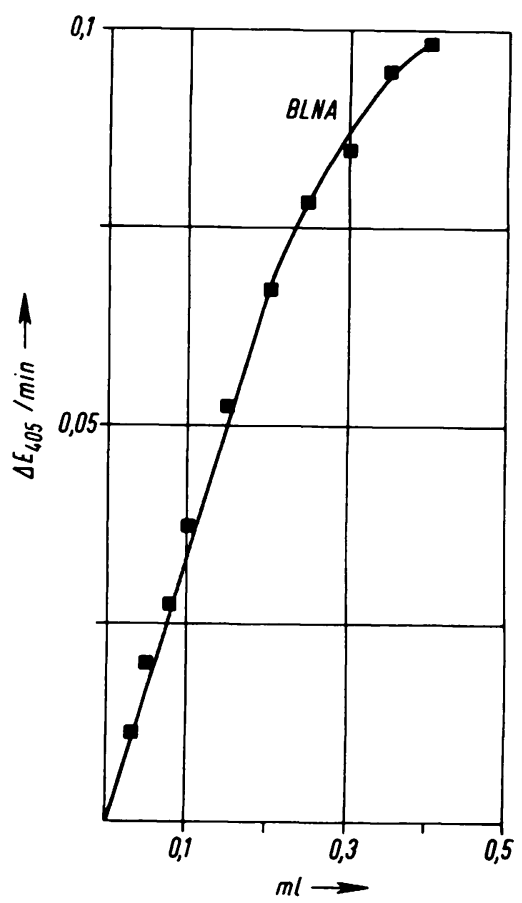




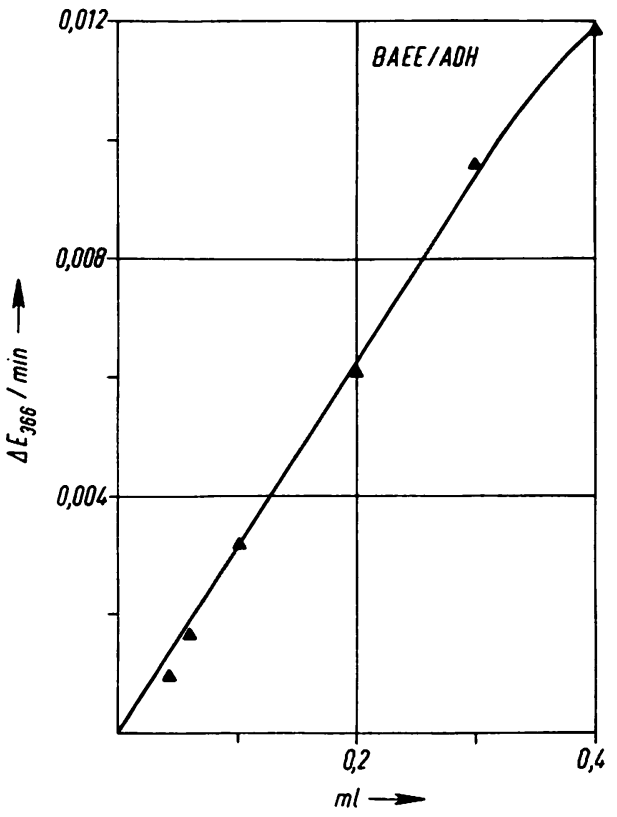

Abb. 3. Spaltung von BAEE durch Eberakrosin.

Ordinate: Extinktionsänderung bei $366 \mathrm{~nm} \mathrm{(ADH/}$ $\mathrm{NAD}^{\oplus}$-System). Abszisse, Bedingungen: s. Legende von Abb. 1 .

min - proportional zur eingesetzten Akrosinmenge zunimmt. Bei der BAPA-Spaltung (Abb. 1) entsprechen dabei $8,3 \mathrm{mU}$, d. i. eine Extinktionsänderung von $0,0276 / \mathrm{min}$, einer $3 \times 10^{-9} \mathrm{M}$ Akrosinkonzentration im Ansatz, das sind $10 \mathrm{pMol}$ Akrosin in der Meßküvette (Endvolumen: 3,0 $\mathrm{ml}$ ). Dieser Wert wurde aus der bei der Titration von Eberakrosin mit dem Trypsin-Plasmin-Inhibitor Bdellin B-3 erhaltenen Titrationskurve ${ }^{[11]}$ berechnet. Die Bestimmung der Aktivitäten derselben Akrosinlösung mit den drei synthetischen Substraten ergab, daß $1 \mathrm{mU}(0,00332 \Delta E / \mathrm{min})$ bei der BLNA-Spaltung $14 \mathrm{mU}(0,0465 \Delta E / \mathrm{min})$ bei der BAPA-Spaltung und $160 \mathrm{mU}(0,176 \Delta E / \mathrm{min})$ bei der BAEE/ ADH-Spaltung entspricht. Für $10 \mathrm{mU}$ bei der BLNA-Spaltung errechnet sich demnach die Akrosinkonzentration im Meßansatz zu $5 \times 10^{-8} \mathrm{M}$ (10 $\mathrm{mU}$ bei der BAPA-Spaltung entsprechen 3,6 $\times$ $10^{-9}$ M Akrosin, s. o.), für $10 \mathrm{mU}$ bei der BAEESpaltung eine solche von nur $3,3 \times 10^{-10} \mathrm{M}$.

Für die Spaltung der Amidsubstrate in $0,2 \mathrm{M}$ Triäthanolamin- $\mathrm{HCl}$ wurden relativ scharfe $\mathrm{pH}$ Optima um 7,8 (BAPA) bzw. 8,0 (BLNA) gefunden Abb. 4).

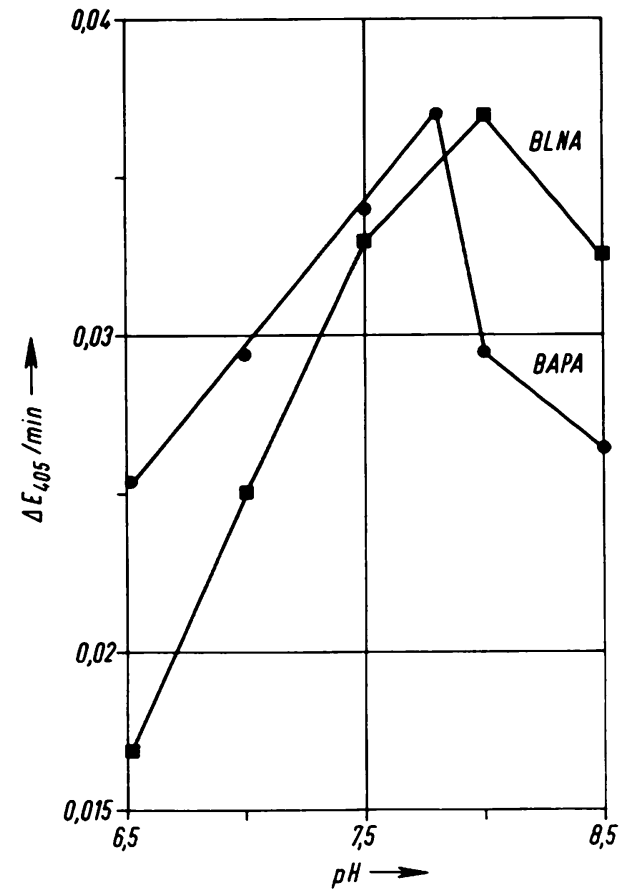

Abb. 4. pH Optima für die Spaltung von BAPA

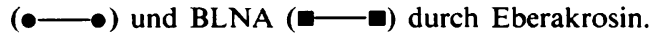

Ordinate: Extinktionsänderung bei $405 \mathrm{~nm}$ pro min. Abszisse: $\mathrm{pH}$-Wert des Meßpuffers (0,2M Triäthanolamin/HCl). Experimentelle Bedingungen: s. „,Material und Methodik"“.

Um Substratsättigung zu erzielen, werden bei den Amidsubstraten relativ hohe molare Konzentrationen benötigt. Die für praktische Zwecke optimale BAPA-Konzentration liegt bei $1 \mathrm{~mm}$ (Abb. 5), die für BLNA bei $2 \mathrm{~mm}$ (Abb. 6). Da die Extinktionszunahme auch bei niedrigeren Substratkonzentrationen (bis $0,25 \mathrm{~mm}$ BAPA und $0,34 \mathrm{~mm}$ BLNA) genügend lange (über $20 \mathrm{~min}$ ) linear verläuft, sind auch unter diesen Bedingungen quantitative Vergleichsmessungen möglich.

Die graphische Ermittlung der Michaeliskonstanten ergab für das System Akrosin/BAPA einen $K_{m}$-Wert von $0,39 \mathrm{~mm}$ (Abb. 5) und für das System Akrosin/BLNA einen solchen von 0,65mм (Abb. 6).

Das Chymotrypsinsubstrat BTEE wurde durch eine Akrosinmenge, die 0,18 U (BAPA) entspricht, nicht gespalten.

\section{Proteinsubstrat (Azocasein)}

Die durch Akrosin verursachte Spaltungsrate von Azocasein, d. h. die Zunahme der Menge der Tri- 
chloressigsäure-löslichen Spaltprodukte im Meßzeitraum, steigt proportional zur eingesetzten Akrosinmenge an: Bei Einsatz von 0,05 ml Akrosinlösung betrug die Extinktionsdifferenz bei $366 \mathrm{~nm}$ gegenüber dem Reagentienleerwert $0,029 \Delta E$, bei $0,1 \mathrm{~m} l 0,057 \Delta E$, bei $0,2 \mathrm{~m} l 0,134 \Delta E$ und bei $0,3 \mathrm{~m} l 0,170 \Delta E$. Auf die BAPA-Spaltung bezogen ergibt sich, daß jeweils $100 \mathrm{mU}$ (BAPA) eine $\mathrm{Zu}$ nahme der Extinktion beim Azocaseintest um $E=$ 0,10 bewirken. Dieselbe Extinktionsänderung wird auch von 1,5 $\mu \mathrm{g}$ Trypsin (Novo Industri $\mathrm{A} / \mathrm{S}$, ca. $70 \%$ rein) hervorgerufen ${ }^{[7]}$.

\section{Synthetische Inhibitoren}

Eberakrosin wird in Gegenwart der irreversiblen Trypsininhibitoren DFP und TLCK ebenfalls inaktiviert, nicht jedoch durch den Chymotrypsininhibitor TPCK. Die graphische Bestimmung der

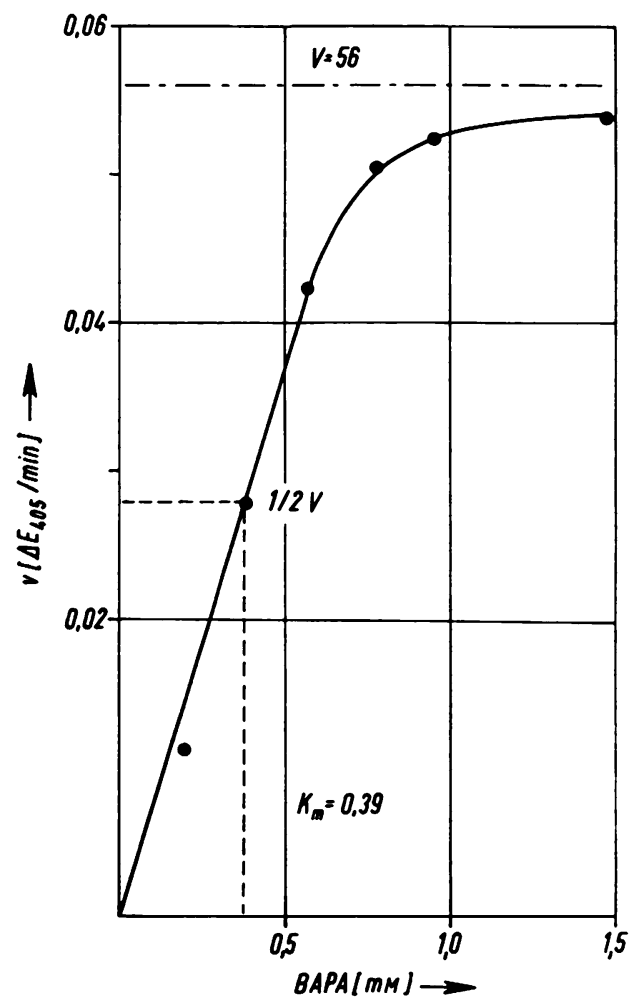

Abb. 5. Bestimmung der Michaeliskonstanten der BAPA-Spaltung durch Eberakrosin.

Ordinate: Extinktionsānderung bei $405 \mathrm{~nm}$ pro min in Abhängigkeit von der BAPA-Anfangskonzentration (Abszisse) im Meßansatz.

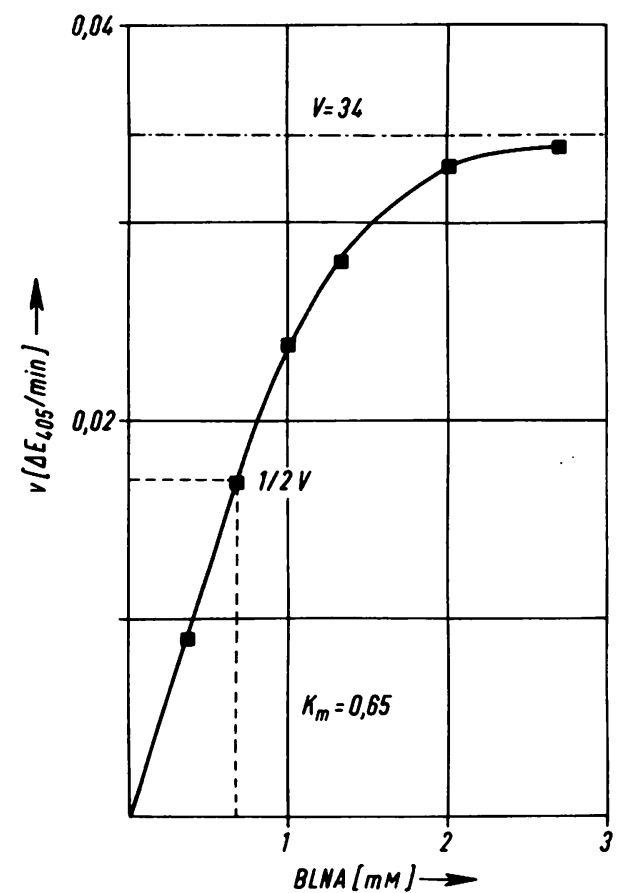

Abb. 6. Bestimmung der Michaliskonstanten der BLNA-Spaltung durch Eberakrosin.

Ordinate und Abszisse: entsprechend Abb. 5.

bimolekularen Geschwindigkeitskonstanten für die Hemmung des Akrosins durch DFP ergab einen Wert von $630 \mathrm{l} \times \mathrm{Mol}^{-1} \times \mathrm{min}^{-1}$ und für die Hemmung durch TLCK $23 l \times \mathrm{Mol}^{-1} \times \min ^{-1}$ (Abb. 7).

\section{Titration mit NPGB}

Analog dem Trypsin läßt sich auch Eberakrosin mit NPGB titrieren. Bei Einsatz von $0,5 \mathrm{ml}$ Akrosinlösung mit einer Aktivität von 1,07 U (BAPA) wurde ein initialer Extinktionssprung (,initial burst") von $E=0,021$ bei $405 \mathrm{~nm}$ gemessen. Dies entspricht einer Enzymmenge von 0,00115 $\mu \mathrm{Mol}$ im Ansatz bzw. 0,93 mU/pMol Akrosin.

\section{Protein-Proteinase-Inhibitoren}

Die Titrationskurven von Eberakrosin mit dem Breitspezifitätsinhibitor aus Seeanemonen und dem Trypsininhibitor II aus Humansperma sind in Abb. 8 dargestellt. Im Gegensatz zu unseren früheren Untersuchungen ${ }^{[10,11,13]}$ wurde hier BLNA als Substrat verwendet.

13 Fritz, H., Schießler, H., Förg-Brey, B., Tschesche, H. \& Fink, E. (1972) diese Z. 353, $1013-1014$. 


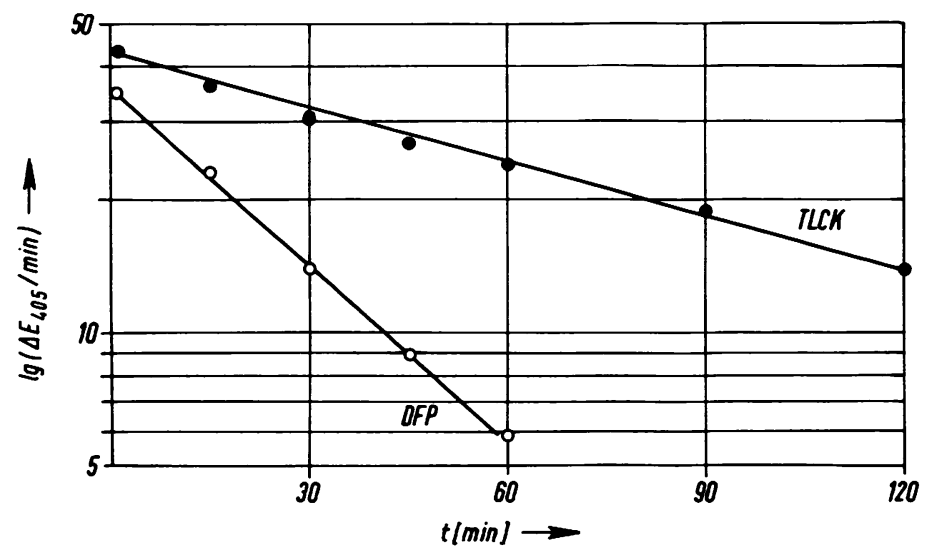

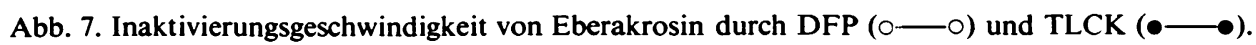

Ordinate: Logarithmus der Extinktionsänderung bei $405 \mathrm{~nm}$ pro min. Abszisse: Inkubationszeit in min.

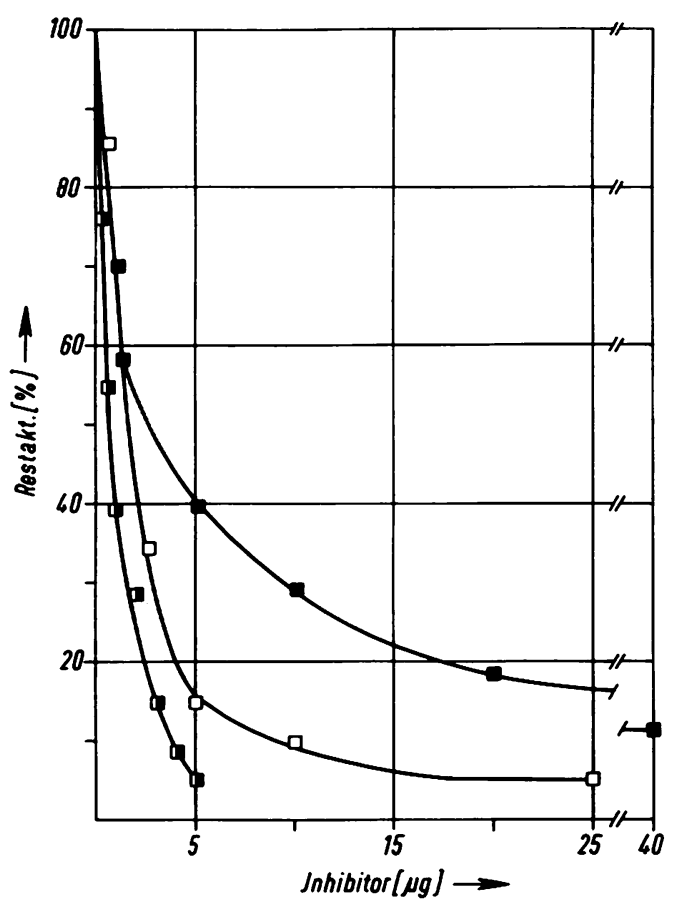

Abb. 8. Titration von Eberakrosin mit Protein-Proteinase-Inhibitoren und Benzamidin bei Verwendung von BLNA als Substrat.

Trypsininhibitor II aus Humansperma: $\mathbf{}-\mathbf{\square}$, Inhibitor aus Seeanemonen: $\square \longrightarrow \square$, Benzamidin: $\square$. Ordinate: Verbleibende Enzymaktivität in \% nach Inkubation des Akrosins mit der auf der Abszisse angegebenen Menge Inhibitor. Zur Versuchsdurchführung s. I. c. ${ }^{[10]}$ sowie ,Material und Methodik" (bezüglich des BLNA-Substrates).

\section{Kompetitive synthetische Hemmstoffe}

Der kompetitive Trypsinhemmstoff Benzamidin besitzt auch eine ausgesprochen hohe Affinität zum Eberakrosin (Abb. 8). Ein Hemmeffekt von pNitroanilin und L-Arginin auf die BAPA-spaltende Aktivität des Akrosins ist erst bei relativ hohen Mengen dieser Substanzen ersichtlich.

\section{Diskussion}

Bei der quantitativen Bestimmung der Aktivitäten von Akrosinlösungen, die bei der Gelfiltration oder Affinitätschromatographie erhalten wurden ${ }^{[4]}$, zeigte es sich, daß vor allem im Bereich niedriger Aktivitäten keine Proportionalität zwischen der eingesetzten Menge an Akrosinlösung und der zu erwartenden Spaltungsaktivität (Substrat: BAPA und BAEE/ADH) vorhanden war. Wurden Verdünnungen hergestellt, so war die gemessene Aktivität jeweils wesentlich niedriger (oft sogar Null!) als die erwartete. Erst die ausschließliche Verwendung von Plastikgefäßen, Plastikpipetten und silikonisierten Küvetten, also die Vermeidung jeglichen Kontaktes mit Glasoberflächen, ermöglichte die Aufstellung der in den Abb. 1 und 3 dargestellten Aktivitätskurven. Die Verluste an Akrosinaktivität durch Oberflächeneffekte sind dabei in sauren Akrosinlösungen wesentlich geringer als im $\mathrm{pH}$ Bereich 7-9. Der Verlust von 95-80\% der Akrosinaktivität innerhalb $1 \mathrm{~min}$ nach der Umstellung des $\mathrm{pH}$-Wertes vom Sauren ins leicht Alkalische des Meßsystems, den Zaneveld und Mitarb. ${ }^{[2]}$ beim Kaninchenakrosin beobachteten, dürfte ebenfalls 
auf die rasch erfolgende Adsorption (Inaktivierung) des Akrosins an der Küvettenoberfläche zurückzuführen sein. Eine so weitgehende Selbstverdauung innerhalb kürzester Zeit bei der vorliegenden geringen Akrosinkonzentration kann man praktisch ausschließen. Die Aktivität von konzentrierteren und verdünnteren Eberakrosinlösungen nimmt bei Inkubation im $\mathrm{pH}$-Bereich $7-8$ bei Zimmertemperatur und $0-4{ }^{\circ} \mathrm{C}$ nach anfänglicher Konstanz nur relativ langsam ab, so $\mathrm{z}$. B. in 3 Tagen bei $0-$ $4{ }^{\circ} \mathrm{C}$ um ca. $30 \%$. Die Aktivität saurer Akrosinlösungen verändert sich, in Übereinstimmung mit den Angaben von Zaneveld und Mitarb. ${ }^{[2,3,14]}$, im Laufe mehrerer Wochen nicht.

Der Zusatz von $\mathrm{CaCl}_{2}$ hatte keinen Einfluß auf die Aktivität des Eberakrosins. Evtl. liegt hier ein speziesbedingtes unterschiedliches Verhalten vor, oder die Adsorption des Kaninchenakrosins ${ }^{[2]}$ sowie des Humanakrosins ${ }^{[3]}$ an Glasoberflächen ist in Gegenwart von Calciumionen vermindert.

Eberakrosin besitzt im Vergleich zu Trypsin und anderen Trypsin-ähnlichen Proteinasen eine extrem hohe Spaltungsaktivität gegenüber BAEE und BAPA. Aus den im Ergebnisteil angegebenen Daten errechnet sich pro Mol Akrosin eine Umsatzrate von ca. $8 \times 10^{8} \mu \mathrm{Mol}$ BAPA pro min bzw. ca. $8 \times 10^{9} \mu \mathrm{Mol}$ BAEE pro min. Diese Werte basieren auf der bei der Titration des Eberakrosins mit dem Trypsin-Plasmin-Inhibitor Bdellin B-3[11] sowie den bei der NPGB-Titration gefundenen molaren Akrosinkonzentrationen, die eine sehr gute Übereinstimmung aufweisen. Allerdings muß der bei der NPGB-Titration erhaltene Wert unter Einsatz größerer Akrosinmengen, die uns vorerst noch nicht zur Verfügung standen, bestätigt werden. Bei den extrem niedrigen molaren Akrosinkonzentrationen in den Testansätzen (BAPA- und BAEE/ADH-System) sind auch die durch Adsorption verursachten Akrosinverluste nicht ungewöhnlich.

Sofern genügend Material zur Verfügung steht, ist die Verwendung des BLNA-Substrates zur Akrosinaktivitätsbestimmung den anderen Substraten gegenüber vorzuziehen. Die Reproduzierbarkeit ist hier besonders gut, da bei den höheren zum Einsatz kommenden Enzymmengen der Einfluß der Adsorptionseffekte entsprechend geringer ist. Zur Aufnahme von Titrationskurven mit Protein-Proteinase-Inhibitoren und kompetitiven Hemm-

14 Polakoski, K. L., Zaneveld, L. J. D. \& Williams, W. L. (1972) Biol. Reprod. 6, 23-29. stoffen ist BLNA als Substrat besonders dann geeignet, wenn die Assoziationskonstanten der betreffenden Akrosin-Inhibitor-Komplexe nicht extrem hoch sind. Die Hemmwirkung der betreffenden Inhibitoren auf Akrosin ist dann wesentlich besser ersichtlich (s. Abb. 8) als z. B. bei Verwendung des BAPA-Substrates ${ }^{[10,11,13]}$ da die Komplexe unter den Bedingungen des BLNA-Testes weniger stark dissoziiert sind.

Die in Spermienextrakten nachgewiesene chymotrypsinähnliche Aktivität (Substrate ATEE[15] und BTEE ${ }^{[16)}$ ist, nach unseren und den Ergebnissen von Zaneveld und Mitarb. ${ }^{[2]}$ zu urteilen, nicht dem Akrosin zuzuschreiben.

Gute Substrate für die Akrosine sind außer BAPA, BLNA und BAEE[2,3] auch $N^{\alpha}$-Tosyl-L-argininmethylester ${ }^{[2,3]}$ und einige weitere synthetische Argininderivate ${ }^{[17,18]}$. Die pH-Optima für die Spaltung dieser Substrate liegen durchwegs nahe 8. Ob die von mehreren Autoren ${ }^{[17,19,20]}$ gefundenen zusätzlichen Spaltungsoptima unter pH 7 ebenfalls auf die Wirkung des Akrosins zurückzuführen sind, ist schwer zu beurteilen. Da diese Autoren für ihre Untersuchungen nur neutrale bis schwach alkalische Spermienextrakte verwendeten, ist mit einer Überlagerung von Enzymfreisetzung durch eine verstärkte Dissoziation der zumindest teilweise vorliegenden Akrosin-Inhibitor-Komplexe bei niedrigeren $\mathrm{pH}-$ Werten und Enzyminaktivierung zu rechnen.

Die Akrosine sind analog wie Trypsin Endoproteinasen. Sie spalten Casein, Ribonuclease ${ }^{[2]}, \mathrm{Fi}-$ brinogen und Gelatine ${ }^{[3]}$. Besonders eindrucksvoll konnte die eiweißspaltende, wahrscheinlich auf Akrosin zurückzuführende Wirkung einzelner Spermien von Gaddum und Blandau ${ }^{[21]}$ demonstriert werden. Aus der starken Hämoglobinspaltung

15 Waldschmidt, M., Karg, H. \& Hoffmann, B. (1964) Naturwissenschaften 51, 18.

16 Stambaugh, R. \& Buckley, J. (1970) Biol. Reprod. 3. 275-282.

17 Multamäki, S. \& Niemi, M. (1972) Int. J. Fertil. 17. 43-52.

18 Ho, J. J. \& Meizel, S. (1970) J. Reprod. Fert. 23, $177-179$.

19 Garner, D. L., Salisbury, G. W. \& Graves, Ch. N. (1971) Biol. Reprod. 4, 93-100.

20 Allison, A. C. \& Hartree, E. F. (1970) J. Reprod. Fert. 21, $501-515$.

21 Gaddum, P. \& Blandau, R. J. (1970) Sciene 170 , $749-751$. 
akrosomaler Extrakte bei pH 3,5 und 4,5[17] bzw. 3,9 und $5,3^{[20]}$ muß gefolgert werden, $d a ß$ noch andere lysosomale Proteasen im Spermienakrosom lokalisiert sind. Um Kathepsin B und $C$ handelt es sich dabei offensichtlich nicht ${ }^{[20]}$. Die Endoproteinaseaktivität zumindest des Eberakrosins ist jedoch wesentlich weniger stark ausgeprägt als beim Trypsin, wenn man auf die Spaltungsaktivitäten gegenüber den synthetischen Substraten bezieht. Eberakrosin dürfte hier eine Mittelstellung zwischen sehr hochspezifischen Enzymen wie Thrombin und den Kallikreinen auf der einen Seite und den Serinproteinasen des Pankreas auf der anderen Seite einnehmen.

Die enge Verwandtschaft des Eberakrosins zum Trypsin ist besonders gut durch die Ergebnisse der Inaktivierungistudien mit DFP und TLCK belegt. Die für die bimolekularen Geschwindigkeitskonstanten der Akrosininaktivierung durch DFP und TLCK ermittelten Werte liegen sehr nahe an denen, die unter denselben Bedingungen für die Inaktivierung von Trypsin gefunden wurden ${ }^{[22]}$. Damit wird der trypsinähnliche Wirkungsmechanismus des Akrosins nicht nur aus qualitativer ${ }^{[2,3,23]}$, sondern auch aus quantitativer Sicht belegt.

Bei kritischer Würdigung der skizzierten Ergebnisse ist zu beachten, daß das zu den Messungen eingesetzte Akrosin wahrscheinlich ein Gemisch von Isoenzymen darstellt. Dies ist aufgrund der mit elektrophoretischen Methoden erhaltenen $\mathrm{Er}$ gebnisse (allerdings unter Verwendung akrosomaler Extrakte) wahrscheinlich ${ }^{[17-19]}$. Da sich Isoenzyme in ihrer Spaltungsaktivität gegenüber Substraten sowie in ihrer Affinität zu Inhibitoren $u$. U. beträchtlich unterscheiden, sind weitergehende kinetische Messungen erst nach Vorliegen einheitlicher Akrosinfraktionen vorgesehen.

Mit Mitteln des Sonderforschungsbereiches 51 finanziert. Herrn Prof. Dr. Dr. E. Werle danken wir für seine Unterstützung, Herrn Dorent Dr. H. Tschesche für den zur Verfügung gestellten Arbeitsplatz (für $\mathrm{H}$. Schießler).

22 DFP: Fahrney, D. E. \& Gold, A. M. (1963) J. Amer. Chem. Soc. 85, 997. TLCK: Shaw, E., Mares-Guia, M. \& Cohen, H. (1965) Biochemistry 4, 2219-2224.

${ }^{23}$ Polakoski, K. L., Williams, W. L. \& McRorie, R. A. (1972) Fed. Proc. 31, 278. 